\title{
"Superdominant" Left Anterior Descending Artery Continuing as Posterior Descending Artery: Extremely Rare Coronary Artery Anomaly
}

\author{
Shakil Sattar Ahmed Shaikh", b, Kalyan Munde ${ }^{a}$, Vishal Patil ${ }^{a}$, Mukund Phutane ${ }^{a}$, \\ Rahul Singla ${ }^{a}$, Zahidullah Khan ${ }^{a}$, Narender Omprakash Bansal ${ }^{a}$
}

\begin{abstract}
The posterior descending artery (PDA) supplying the posterior onethird of the inter-ventricular septum usually arises from the right coronary artery (RCA) or the left circumflex artery (LCX). PDA arising from the left anterior descending artery (LAD) is an extremely rare anomaly. Here we report a rare type of left dominant circulation in which a large LAD is continuing as PDA after winding round the apex in the presence of a diminutive RCA. Such a large LAD continuing as PDA is referred as "hyperdominant" or "superdominant". A 32-yearold male chronic smoker presented with acute onset retrosternal pain of $4 \mathrm{~h}$ duration with profuse sweating in primary health center with electrocardiography (ECG) changes in inferior leads and was thrombolysed with intravenous streptokinase 15 lacs IU over one hour and was referred to our center for further management and coronary intervention. Coronary angiogram revealed PDA as a continuation of the LAD beyond the crux and a non-dominant right coronary as well as LCX. The LAD had plaque in mid-LAD course. Intravascular ultrasound study (IVUS) showed insignificant plaque in mid-LAD (30\%). Hence, we decided to keep him on medical therapy only.
\end{abstract}

Keywords: Hyperdominant left anterior descending artery; Posterior descending artery; Coronary artery anomaly

\section{Introduction}

No two coronary anatomic patterns are alike and there is a wide range of variability within the normal distribution. Coronary artery anomalies represent marked deviations from normal but fortunately, have a relatively constant incidence of less than $1.5 \%$ across different racial groups [1]. It could be

Manuscript submitted June 3, 2018, accepted June 12, 2018

aDepartment of Cardiology, Grant Medical College, Sir JJ Group of Hospital, Mumbai, India

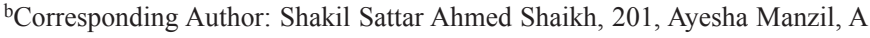
Wing Sunrise Galaxy Housing Society, Dr. Ambedkar Road, Kalyan (west), Maharashtra, India. Email: drshakilsshaikh@rediffmail.com

doi: https://doi.org/10.14740/cr738w considered an incidental finding without clinical significance. However, depending upon the origin, course and termination of the anomalous vessel, these abnormalities may be responsible for angina pectoris, heart failure and sudden death [1]. Posteroinferior part of the muscular interventricular septum is normally supplied by posterior descending artery (PDA) which may arise from right coronary artery (in right dominance or codominance pattern) or from LCX (in left dominance pattern) [2]. Supply of the posteroinferior septum by a hyperdominant left anterior descending artery (LAD) continuing as PDA is extremely rare and sporadically reported in the literature [6]. We describe here the clinical presentation and angiographic findings of a patient with inferior wall myocardial infarction with coronary angiogram which revealed plaque in mid-LAD with PDA arising as the continuation of the LAD in the presence of a non-dominant right coronary artery (RCA).

\section{Case Report}

A 32-year-old male chronic smoker, non-hypertensive and nondiabetic, presented with acute onset retrosternal pain of 4-h duration with profuse sweating in primary health center (Table 1). On examination, pulse was $80 / \mathrm{min}$ and blood pressure was 120/70 $\mathrm{mm} \mathrm{Hg}$. Electrocardiography (ECG) revealed ST segment elevation in inferior leads (Fig. 1). Patient was thrombolysed with intravenous streptokinase 15 lacs IU over one hour and then referred to tertiary care center for further management and coronary intervention. Patient laboratory parameters showed elevated cardiac enzymes (Table 2). Chest radiograph showed no abnormality (Fig. 2). Echocardiography revealed basal inferoseptal and inferior wall hypokinesia with left ventricle ejection fraction of $45 \%$. Coronary angiogram revealed PDA as a continuation of the LAD beyond the crux (Fig. $3 \& 4$ ) and the RCA, although normal in origin, was diminutive and terminated on the lateral wall of the right ventricle (Fig. 5). LCX artery was also non-dominant. The LAD had plaque in mid-LAD course. Intravascular ultrasound study (IVUS) showed insignificant plaque (30\% lesion) in mid LAD. Hence, we decided to keep him on medical therapy.

\section{Discussion}

Most coronary anomalies do not result in signs, symptoms or 
Table 1. Timeline of Patient Presentation

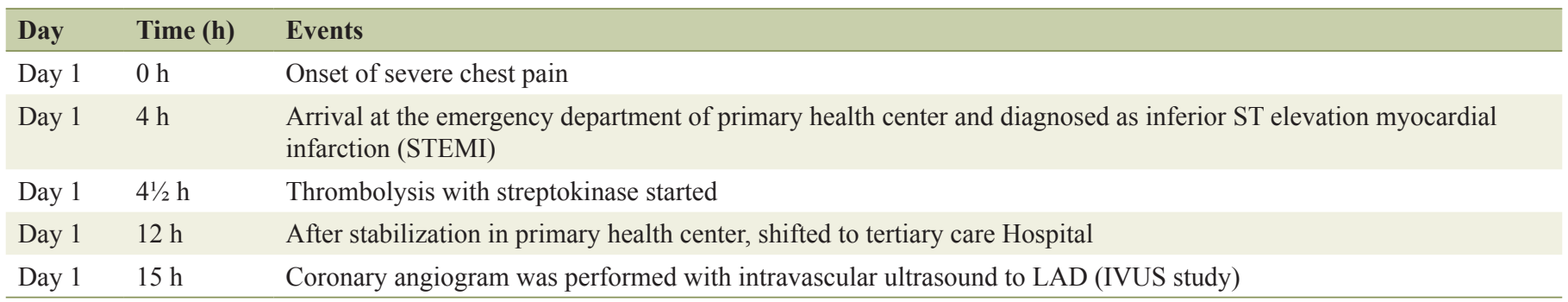
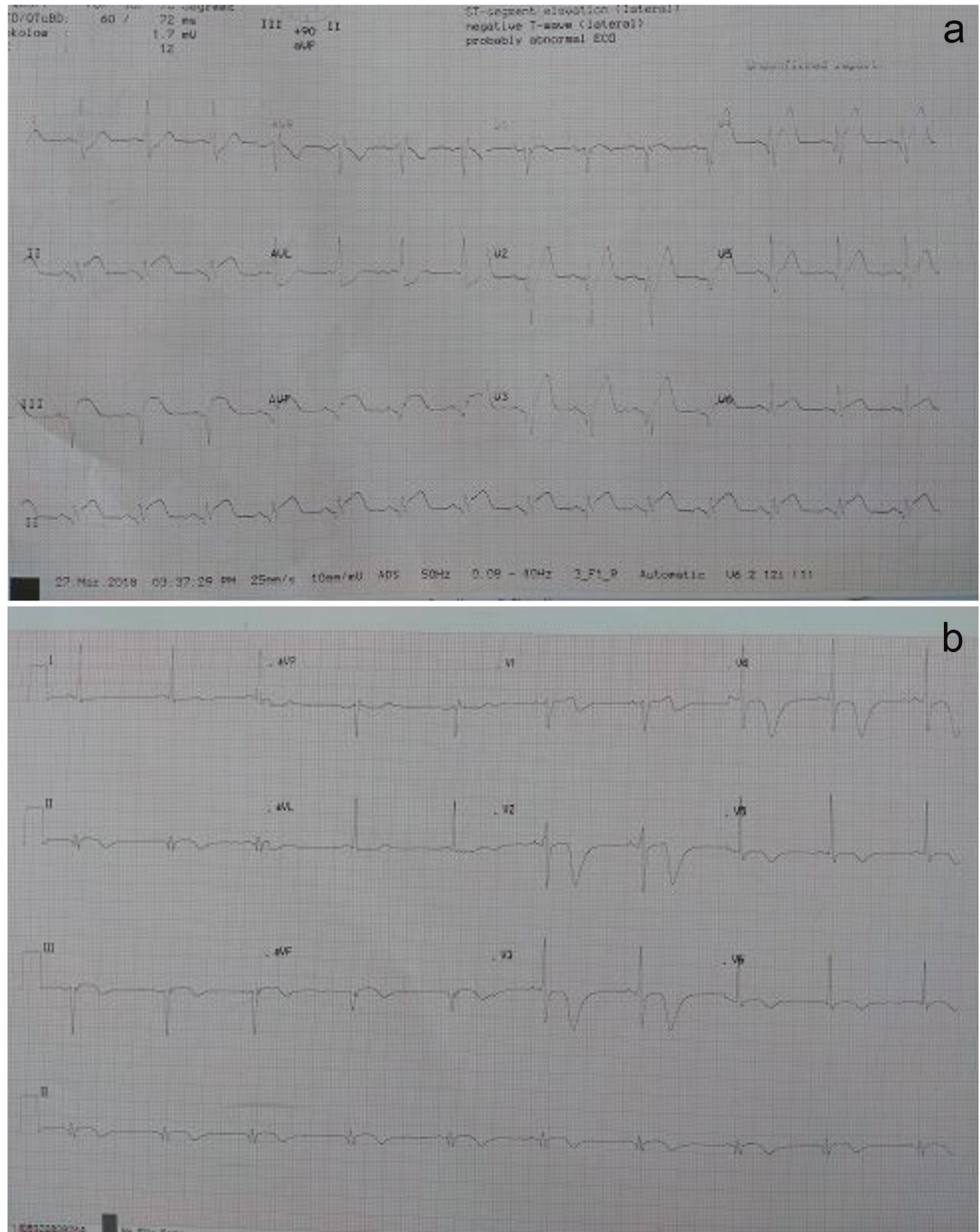

Figure 1. (a) 12-lead ECG showing ST segment elevation in leads II, III and aVF with T wave changes in chest leads; (b) 12-lead ECG after thrombolysis with streptokinase. There is ST segment resolution with q wave in leads III and AVF with T wave changes in chest leads. 
Table 2. Laboratory Profiles of Patient

\begin{tabular}{lll}
\hline Lab parameter & Patient value & Reference range \\
\hline Hemoglobin & $13.2 \mathrm{gm} / \mathrm{dL}$ & $12-14 \mathrm{gm} / \mathrm{dL}$ \\
Total white blood cell count & $11.36 \times 10^{9} / \mathrm{L}$ & $4 \times 10^{9}-10 \times 10^{9} / \mathrm{L}$ \\
Platelet count & $187 \times 10^{9} / \mathrm{L}$ & $100 \times 10^{9}-300 \times 10^{9} / \mathrm{L}$ \\
Troponin I & $0.76 \mathrm{ng} / \mathrm{mL}$ & $0-0.2 \mathrm{ng} / \mathrm{mL}$ \\
Creatine kinase-MB & $14.53 \mathrm{ng} / \mathrm{mL}$ & $0.10-4.94 \mathrm{ng} / \mathrm{mL}$ \\
Total cholesterol & $4.8 \mathrm{mmol} / \mathrm{L}$ & $<5.0 \mathrm{mmol} / \mathrm{L}$ \\
HDL & $0.82 \mathrm{mmol} / \mathrm{L}$ & $>1.0 \mathrm{mmol} / \mathrm{L}$ \\
LDL & $2.84 \mathrm{mmol} / \mathrm{L}$ & $<3.0 \mathrm{mmol} / \mathrm{L}$ \\
Triglycerides & $1.63 \mathrm{mmol} / \mathrm{L}$ & $<1.7 \mathrm{mmol} / \mathrm{L}$ \\
HbAlc & $5.9 \%$ & $5.5-6.5 \%$ \\
\hline
\end{tabular}

complications, and usually are discovered as incidental findings at the time of catheterization, autopsy or other radiological investigations. According to the literature, the incidence of coronary artery anomalies is less than $1.5 \%$ [1]. Based on that, the concept of coronary artery dominance was proposed by Schlesinger. Right dominance (in $85 \%$ of patients) means that PDA, atrioventricular (AV) nodal artery and the posterolateral branches, all arise from the RCA. Left dominance (in $8 \%$ of cases) means that all three vessels (PDA, AV nodal and the posterolateral branches) arise from LCX artery. Co-

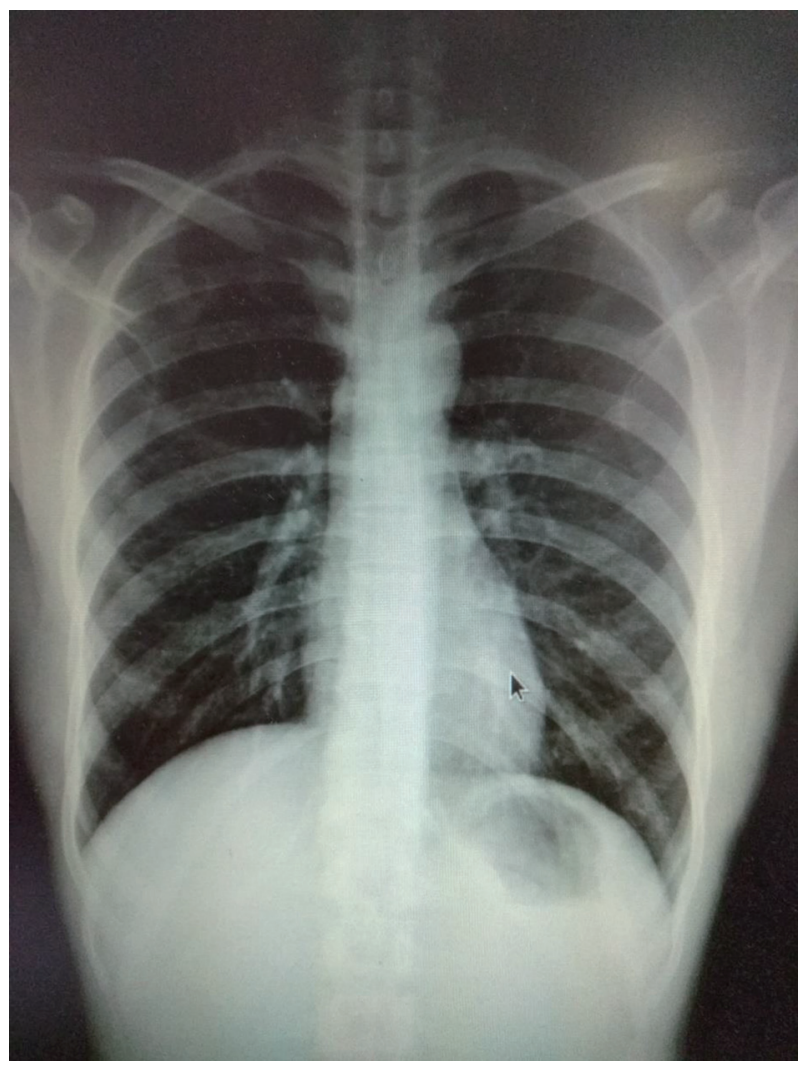

Figure 2. Chest radiograph (posteroanterior view) shows no significant abnormality. dominance (in 7\% of cases) is seen when PDA arises from the LCX artery and the posterolateral branches arise from the $\mathrm{RCA}$ [2]. Several unusual variations in the origin of PDA have been reported such as dual PDA, early origin of the PDA from the RCA, PDA from an obtuse marginal or septal perforator artery, perfusion of the inferior septum by right ventricular branches or acute marginal artery and other variations including PDA arising from a vascular ring connecting RCA and LCX [3]. It is rare for the PDA to originate from the LAD, even though continuation of the LAD around the apex is commonly seen, usually referred as "wrap around" LAD [4]. Occurrence of acute inferior wall myocardial ischemia due to occlusion of LAD continuing as PDA is also reported [5]. Clark et al described three patients whose PDA originated from the LAD and in all, the PDA terminated before the crux [6]. None

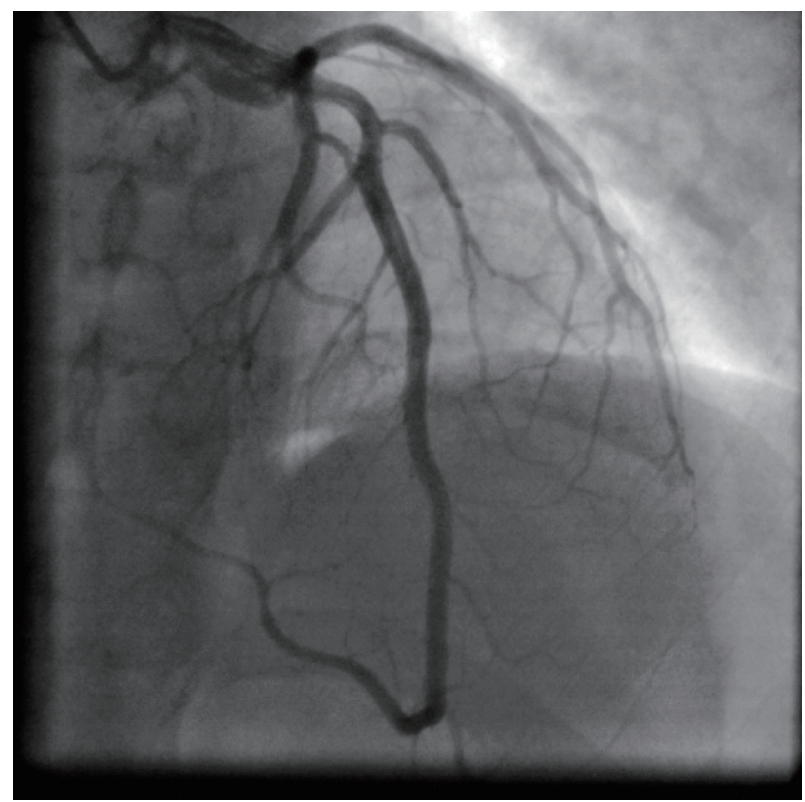

Figure 3. Coronary angiogram showing left anterior descending artery (LAD) extending into the posterior interventricular groove. Note that the LAD wraps around the apex and gives rise to an anomalous large posterior descending artery (PDA). 


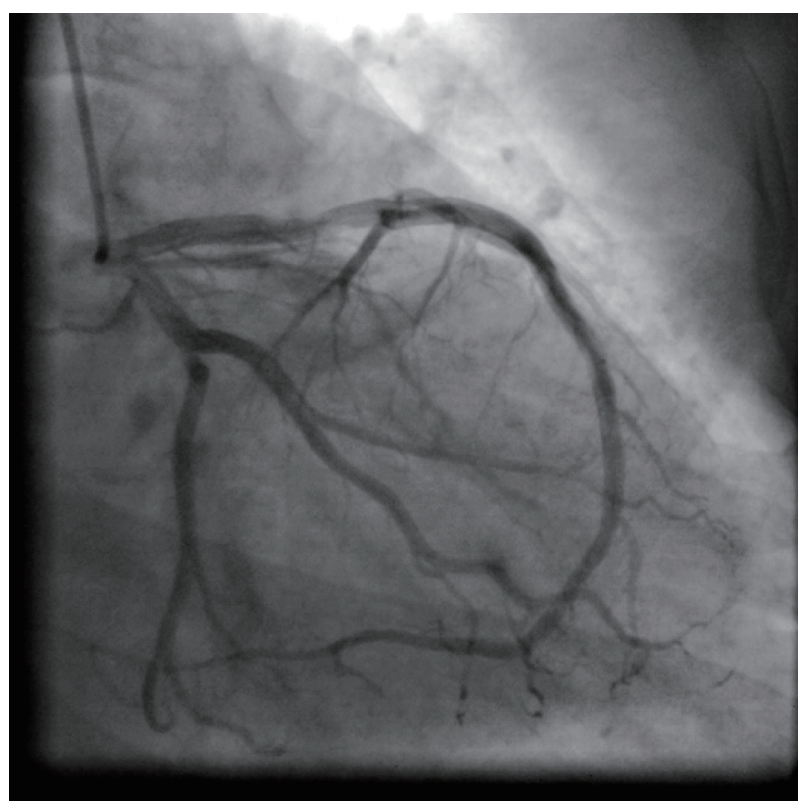

Figure 4. Coronary angiogram in the right anterior oblique (RAO) caudal projection showing left anterior descending artery continuing as posterior descending artery with non-dominant left circumflex artery.

of these PDA extended beyond the crux to continue in the posterior interventricular groove. Musselman et al was the first to describe PDA arising from the LAD and extending beyond the crux. However, the artery then gave two branches travelling on either side of the posterior interventricular sulcus and gave an AV nodal branch as well [7]. Javangula et al described the first patient in world literature with LAD continuing as PDA across the left ventricular apex in the presence of a normally situated right coronary ostium with an atretic small RCA and coexistent aortic stenosis .However, in their report, the LAD continued as the PDA up to the crux of the heart [8]. However, in our case, the LAD was hyperdominant in the true sense that it did travel for a considerable distance into the posterior interventricular sulcus beyond the crux as the PDA and the RCA were non-dominant. To the best of our knowledge, among reported cases it is an extremely rare case worldwide in which the hyperdominant LAD continued as PDA beyond the crux into the posterior interventricular sulcus.

\section{Conclusions}

The clinical importance of this anomaly is that if superdominant LAD is occluded, it will cause a massive infarct affecting the anterior wall, septum and inferior wall and can lead to cardiogenic shock with high morbidity and mortality without timely management and intervention. Physicians, interventional cardiologists and cardiac surgeons should be aware of such a rare anomaly as it has a considerable impact on clinical outcome of a patient with occluded "hyperdominant" LAD jeopardizing a large segment of the myocardium. Our case report and review of literature will help in its early diagnosis and planning better management strategies.

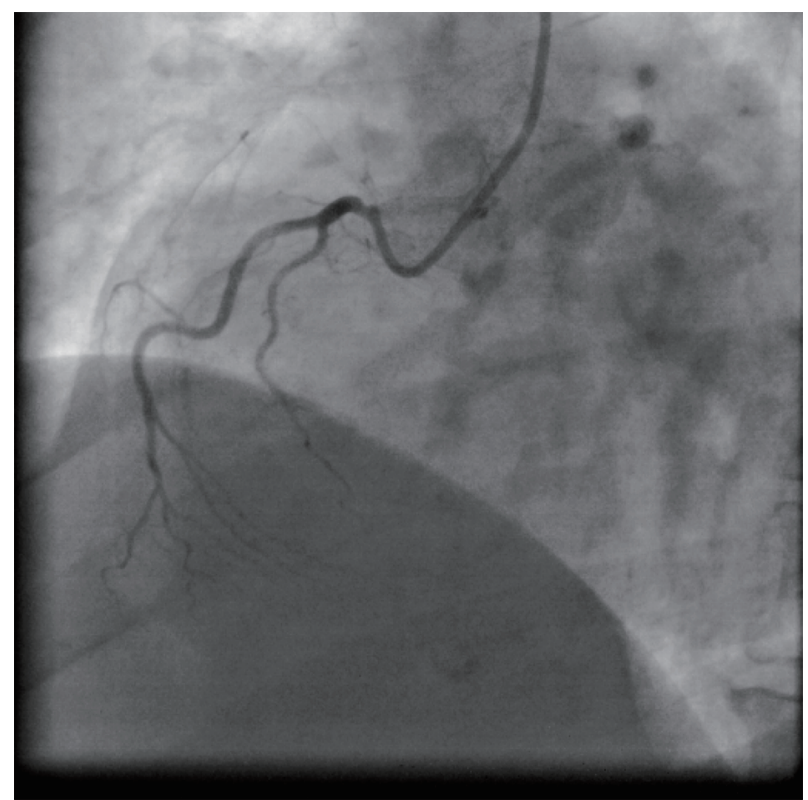

Figure 5. Coronary angiogram in the left anterior oblique (LAO) cranial projection showing that rudimentary (non-dominant) right coronary artery originates from the right coronary cusp.

\section{Acknowledgments}

We are grateful to Dr Narender O. Bansal, Professor and Head of Department of Cardiology, Sir JJ Group of Hospital, Mumbai, India for allowing us to publish this case report.

\section{Conflict of Interest}

None.

\section{Guarantor}

Dr Shakil Sattar Ahmed Shaikh will act as the guarantor of the manuscript.

\section{Financial Support}

None.

\section{References}

1. Yamanaka O, Hobbs RE. Coronary artery anomalies in 126,595 patients undergoing coronary arteriography. Cathet Cardiovasc Diagn. 1990;21(1):28-40.

2. Schlesinger MJ. Relation of anatomic pattern to pathologic conditions of the coronary arteries. Arch Pathol. 1940;30:403-415.

3. Kim JH, Cha KS, Park SY, Park TH, KimMH, Kim YD. 
Anomalous origins of the right and posterior descending coronary arteries from the left anterior descending coronary artery: unusual pattern of single coronary artery. J Cardiol Cases. 2011;3(1):e26-e28.

4. Ilia R, Weinstein JM, Wolak A, Gilutz H, Cafri C. Length of left anterior descending coronary artery determines prognosis in acute anterior wall myocardial infarction. Catheter Cardiovasc Interv. 2014;84(2):316-320.

5. Sunil Roy TN, Nagham JS, Kumar RA. Acute inferior wall myocardial infarction due to occlusion of the wrapped left anterior descending coronary artery. Case Rep Cardiol. 2013;2013:983943.
6. Clark VL, Brymer JF, Lakier JB. Posterior descending artery origin from the left anterior descending: an unusual coronary artery variant. Cathet Cardiovasc Diagn. 1985;11(2):167-171.

7. Musselman DR, Tate DA. Left coronary dominance due to direct continuation of the left anterior descending to form the posterior descending coronary artery. Chest. 1992;102(1):319-320.

8. Javangula K, Kaul P. Hyperdominant left anterior descending artery continuing across left ventricular apex as posterior descending artery coexistent with aortic stenosis. J Cardiothorac Surg. 2007;2:42. 